\title{
In Vitro and In Vivo Effect of Acylated and Unacylated Ghrelin on Neonatal Glucose Homeostasis
}

\author{
HEHONG NI, KATHLEEN DE WAELE, PALLAVI WALIA, AND JEAN-PIERRE CHANOINE
}

\begin{abstract}
Endocrinology and Diabetes Unit, British Columbia Children's Hospital, University of British Columbia, Vancouver, British Columbia
\end{abstract} V6N 3V4, Canada

\begin{abstract}
Maintenance of normal glucose homeostasis is crucial for survival during the perinatal period. Acylated ghrelin (AG) but not unacylated ghrelin (UAG) inhibits insulin release from pancreatic islets in adult rats. Circulating AG concentrations are low in the fetus and progressively increase in the postnatal period. We tested the hypothesis that AG has insulinostatic effects in vitro and in vivo during the perinatal period. In vitro, AG $\left(10^{-10}-10^{-8} \mathrm{M}\right)$ caused a $25-53 \%$ decrease in insulin secretion by islets from 5-d-old rat pups under normo- and hyperglycemic conditions, an effect that was mediated through the growth hormone secretagogue receptor (GHSR1a). Ghrelin (1-5) amide, [Dap3]-octanoyl, a pentapeptide that is resistant to deacylation and binds the GHSR-1a, had similar effects at $10^{-8}$ M. In vivo, AG, UAG, or GHRP-6 [D-Lys3], a GHSR-1a antagonist, did not affect insulin or glucagon concentrations during the first $3 \mathrm{~h}$ of life. In 6-d-old pups, AG, UAG, or ghrelin (1-5) amide, [Dap3]-octanoyl did not affect glucose-induced insulin or C-peptide concentrations. In summary, AG has insulinostatic effects in vitro as early as during the perinatal period. These effects could not be confirmed in vivo, possibly due to the short half-life of AG in rat neonates. (Pediatr Res 67: 609-613, 2010)
\end{abstract}

$\mathrm{G}$ hrelin is a peptide hormone mainly secreted by the stomach in adult rodents and humans. It circulates as acylated ghrelin (AG) and unacylated ghrelin (UAG). Acylation with octanoate is required for binding of ghrelin to the growth hormone $(\mathrm{GH})$ secretagogue receptor (GHSR1-a) that mediates many of the actions ascribed to ghrelin. Originally, AG was considered to be the active form of the hormone and its main roles were felt to be limited to GH secretion (1) and stimulation of appetite at the hypothalamus level (2). It is now clear that AG has many metabolic effects, that UAG is not inactive, and that both $\mathrm{AG}$ and UAG potentially play a physiologic role in energy balance (reviewed in Ref. 3).

The source of ghrelin and its concentrations in tissues and plasma differ according to age. The rat embryo contains elevated plasma concentrations of UAG but little AG (4), and the circulating AG to UAG ratio increases markedly between fetal day 20 and postnatal day (PN) 1 (5). Concentrations of ghrelin are barely detectable in the fetal stomach and increase progressively to reach adult values by $3-4 \mathrm{wk}$. In contrast, the

Received September 14, 2009; accepted January 11, 2010

Correspondence: Jean-Pierre Chanoine, MD, PhD, Endocrinology and Diabetes Unit, Room K4-212, British Columbia's Children's Hospital, 4480 Oak Street, Vancouver BC V6H 3V4 Canada; e-mail: jchanoine@cw.bc.ca

Supported in part by grant MOP 81291 of the Canadian Institutes of Health and Research (CIHR) and by grant 1637 from the Canadian Diabetes Association (CDA) to J.-P.C. K.D. was the recipient of a ESPE research fellowship. fetal pancreas contains elevated concentrations of ghrelin that progressively decrease after birth (5). In pancreas, ghrelin originates from glucagon producing alpha $(\alpha)$ cells but also from a novel cell type, called epsilon $(\epsilon)$. Insulin-producing beta $(\beta)$ cells do not contain ghrelin (6). In adult pancreas, GHSR-1a is present on the $\alpha$-cell as well as on the $\beta$-cell (7), raising the possibly of a direct, paracrine action of ghrelin on insulin and glucagon secretion.

Indeed, AG has been shown in vitro to inhibit insulin and stimulate glucagon secretion by cultured pancreas islets from adult rodents (rev in Ref. 8). The in vivo role of ghrelin on glucose metabolism remains unclear but endogenous ghrelin secreted by islets was suggested to modulate insulin secretion by the $\beta$-cell in adult rodents (9).

We hypothesized that AG could contribute to neonatal normoglycemia by inhibiting insulin production by the $\beta$-cell. Maintenance of normoglycemia is a requirement for early PN survival in rodents like in most mammals. This is achieved through a marked decrease in insulin and increase in glucagon production and by the development of hepatic glucose production in a matter of minutes following birth (10).

In this study, we investigated the effects of ghrelin (1) in vitro on insulin production by pancreas islets from 5-d-old rats; (2) in vivo on glucose homeostasis in rat neonates born by Cesarian section on the expected day of delivery and in 6-d-old rat pups.

\section{METHODS}

Animals. Sprague Dawley rats obtained from Charles River Laboratories (St. Constant, Quebec, Canada) were time-mated in the animal care center at the Child and Family Research Institute (day of conception was day 0). They had free access to water and to a standard chow diet. Except if otherwise noted, all chemical reagents were obtained from Sigma Chemical Co.-Aldrich Canada (Oakville, ON). All experiments were approved by the University of British Columbia Animal Care Committee.

In vitro studies. Pancreases from 5-d-old rat pups killed by decapitation were removed. Islets were isolated by collagenase XI digestion $(1000 \mathrm{U} / \mathrm{mL}$ in Hanks' solution incubated at $37^{\circ} \mathrm{C}$ for $17 \mathrm{~min}$ with gentle shaking). The suspension was washed in Hank's solution, centrifuged at $1000 \mathrm{rpm}$ for $30 \mathrm{~s}$ at $4{ }^{\circ} \mathrm{C}$ and the supernatant aspirated. This step was performed three times. Islets were then resuspended in RPMI 1640 medium (Invitrogen, Carlsbad, $\mathrm{CA}$ ) and poured on a prewet $70 \mu \mathrm{m}$ nylon mesh cell strainer (BD Biosciences, Mississauga, ON). Islets are hand-picked under a microscope, and incubated overnight in RPMI 1640 medium supplemented with $10 \%$ fetal bovine serum, $100 \mathrm{U} / \mathrm{mL}$ penicillin $\mathrm{G}, 100 \mu \mathrm{g} / \mathrm{mL}$ streptomycin (Invitrogen). After prein-

\footnotetext{
Abbreviations: AG, Acylated ghrelin; CV, Coefficient of variation; GHSR-1a, Growth hormone secretagogue receptor 1a; PN, Postnatal; UAG, Unacylated ghrelin
} 
cubation for $2 \mathrm{~h}$ at $37^{\circ} \mathrm{C}$ with $5 \% \mathrm{CO}_{2}$ in Krebs-Ringer-Bicarbonate-HEPES (KRBH) buffer with $1.67 \mathrm{mM}$ glucose, islets were incubated for $1 \mathrm{~h}$ at $37^{\circ} \mathrm{C}$ in $500 \mu \mathrm{L}$ of the same KRBH buffer in 48 -well plates (five islets per well) under hypoglycaemic $(1.67 \mathrm{mM})$, euglycemic $(8.5 \mathrm{mM})$, or hyperglycaemic conditions (16.7 mM glucose). Half of the cultures were performed in male and half in female pups. The effect of the following chemicals (Phoenix Pharmaceuticals, Burlingame, CA) on insulin secretion by the pancreas islet were tested: rat AG (031-31); rat UAG (031-33); ghrelin (1-5) amide [Dap3]-octanoyl (032-14, a pentapeptide that binds to the GHSR-1a (11)); GHRP-6 [D-Lys3] (031-22, a GSHR-1a antagonist (12)) or [D-Arg1, D-Phe5, D-Trp7,9, Leu11]-substance P (061-21, a GSHR-1a inverse agonist (13)). The supernatant was collected and stored at $-80^{\circ} \mathrm{C}$ until analysis.

In vivo effect of $A G, U A G$, and GHRP-6 [D-Lys3] administration on glucose homeostasis at birth. Twenty-one dams were randomly assigned to a baseline, $45 \mathrm{~min}$ and $180 \mathrm{~min}$ group. All pups were delivered by Cesarian section immediately after decapitation of the dam on the morning of pregnancy day 21.5 , a few hours before the expected time of spontaneous delivery. In the baseline group $(n=6)$, all pups in one litter were killed immediately after delivery. In the 45 min group $(n=8), 3-4$ pups in each litter were randomly assigned to receive at birth an i.p. injection $(50 \mu \mathrm{L})$ of either saline, $1 \mu \mathrm{g} \mathrm{AG}(=0.3 \mathrm{nmol}), 1 \mu \mathrm{g}$ UAG $(=0.3 \mathrm{nmol})$, or a mixture of $1 \mu \mathrm{g} \mathrm{AG}$ and $25 \mu \mathrm{g}(=27 \mathrm{nmol})$ GHRP-6 [D-Lys3] $)$ and were killed $45 \mathrm{~min}$ after the injection. In the $180 \mathrm{~min}$ group $(n=7), 3-4$ pups in each litter were randomly assigned to receive 2 consecutive injections (at birth and after $120 \mathrm{~min}$ ) as described above and were killed at $180 \mathrm{~min}$ of life. Blood from pups receiving the same treatment at the same time point in the same litter was pooled as one sample. Thus, each litter from the six dams in the baseline group contributed one baseline blood sample. Each litter from the eight dams in the $45 \mathrm{~min}$ group and from the seven dams in the 180 min group each contributed one blood sample to the control, one to the AG, one to the UAG and one to the AG/GHRP-6 [D-Lys3] groups. Pups were kept in a warm $\left(33^{\circ} \mathrm{C}\right)$ and humid $(60 \%)$ environment (Ohio Servo-Controlled Isolation Incubator, Aireo, Madison, WI) and were killed by decapitation. Trunk blood was collected in a tube containing EDTA and $250 \mathrm{U}$ aprotinin (Trasylol, Bayer, Toronto, ON) per ml blood. All samples were kept on ice until centrifugation and plasma was frozen at $-80^{\circ} \mathrm{C}$ until hormonal determinations were performed. Previous data from our group have shown that i.p. administration of $1 \mu \mathrm{g} \mathrm{AG}$ or UAG in rat neonates results in a rapid and sustained (at least for $90 \mathrm{~min}$ ) absorption of the peptide into the circulation (14). Intraperitoneal administration of GHRP-6 [D-Lys3] was previously shown to be an effective way of decreasing appetite in rodents, suggesting that it is also readily absorbed (12).

In vivo effect of AG, UAG, ghrelin (1-5) amide [Dap3]-octanoyl, and GHRP-6 [D-Lys3] administration on glucose-induced hyperinsulinemia and C-peptidemia in 6-d-old pups. Eight dams were allowed to deliver normally and the litter culled to 10 pups on PN1 (five male and five female pups). On PN6, each male and female pup in each litter was fasted for $30 \mathrm{~min}$ and randomly assigned to one of the following five groups: glucose only (control group, i.p. injection of $1.4 \mathrm{~g}$ glucose/kg body weight in $100 \mu \mathrm{L}$ ) or glucose plus either AG ( $25 \mathrm{nmol} / \mathrm{kg}$ body weight), UAG ( $25 \mathrm{nmol} / \mathrm{kg})$, ghrelin (1-5) amide [Dap3]-octanoyl $(25 \mathrm{nmol} / \mathrm{kg})$, or a mixture of ghrelin (1-5) amide, [Dap3]-octanoyl $(25 \mathrm{nmol} / \mathrm{kg})$, and GHRP-6 [D-Lys3] $(1 \mu \mathrm{mol} / \mathrm{kg})$. The dose of AG and time of sacrifice were chosen following an experiment previously performed in adult male rats. Eight adult animals were given an i.p. administration of glucose $(1.4 \mathrm{gr} / \mathrm{kg})$ with or without $\mathrm{AG}(25 \mathrm{nmol} / \mathrm{kg})$ in random order at 1 week interval. When glucose was administrated with ghrelin, blood glucose concentrations 15 and $30 \mathrm{~min}$ after the injection were, respectively, $50 \%$ (13.2 versus $9.2 \mathrm{mM}$ ) and $48 \%$ (13.6 versus $9.2 \mathrm{mM}$ ) higher than when glucose was injected alone. The pups were kept in a warm $\left(33^{\circ} \mathrm{C}\right)$ and humid (60\%) environment and fasted for 30 min before i.p. injection. The pups were killed 20 min after the injection and trunk EDTA blood was collected. Blood glucose was measured in duplicate by glucometer. All samples were kept on ice until centrifugation and plasma was frozen at $-80^{\circ} \mathrm{C}$ until determinations of insulin and c-peptide were performed.

Assays. Blood glucose was measured using the One-Touch Ultra glucose meter (Life Scan Canada Ltd, Burnaby, BC, Canada). In the in vivo experiment at birth, insulin (RI-13 K, inter-assay coefficient of variation [CV] $<9.4 \%$, intra-assay $\mathrm{CV}<4.6 \%$ ) and glucagon (GL-32 K, intra-assay CV $4.3 \%$ ) were determined by RIA (Millipore, St-Charles, MO). In the other experiments, insulin was determined by rat specific ELISA (80-INSRT-E01, or 80-INSRTH-E01 [high range], intra-assay CV $<6.4 \%$, inter-assay CV $<9.9 \%$, Alpco, Salem, NH). C-peptide was determined by ELISA (rat specific, recognizes C-peptide I and II, 48-CPERT-E01, intra- and inter-assay CVs $<8.8 \%$, Alpco).

Statistics. Results are expressed as mean (standard error of the mean). The differences between the groups were assessed using ANOVA with posthoc analysis for comparison with the control group using $t$ test for unpaired values. Data were $\log$ transformed for the in vitro experiments. A $p$ value

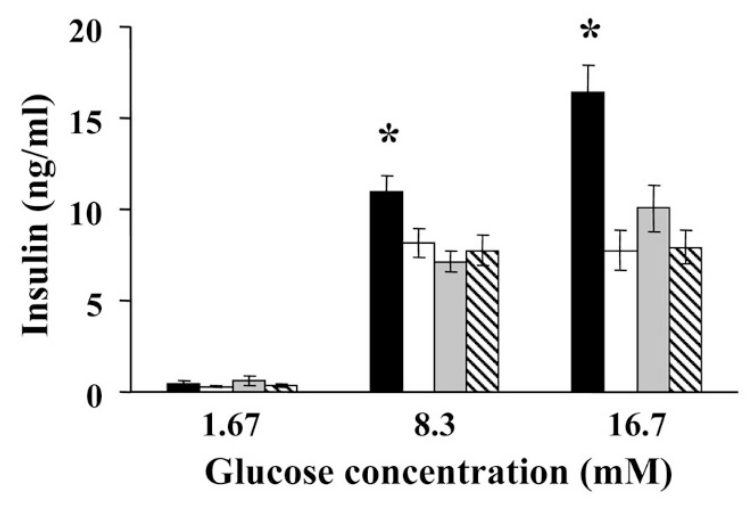

Figure 1. Effect of acylated ghrelin (AG) on insulin secretion by cultured pancreas islets from 5-d-old rats under hypoglycemic $(1.67 \mathrm{mM})$, normoglycemic $(8.3 \mathrm{mM})$, and hyperglycemic $(16.7 \mathrm{mM})$ conditions. $\square$, control; $\square$, AG $0.1 \mathrm{nM} ; \square$, AG $1.0 \mathrm{nM} ; \mathbf{\nabla}$, AG $10 \mathrm{nM}$. Two-way ANOVA: $p<0.001$ for glucose; $p<0.05$ for AG concentration; $p<0.05$ for glucose $\times \mathrm{AG}$ concentration interaction. $* p<0.05$ compared with all other groups. Values are given in mean (SE).

$<0.05$ was considered significant. Data were analyzed with SPSS version 17.0 (2008, Chicago, IL).

\section{RESULTS}

In vitro studies. As expected, the concentration of insulin in the culture medium after incubation of the pancreas islets increased with the concentration of glucose. Addition of AG caused a marked decrease in insulin secretion (ANOVA: $p<$ 0.05 ) that was similar for AG concentrations of $0.1,1$, and 10 $\mathrm{nM}$ and was greater under hyperglycemic $(-39$ to $-53 \%)$ compared with euglycemic conditions ( -25 to $-29 \%$ ) (Fig. 1). In contrast, addition of UAG or of a mixture of AG and UAG did not affect insulin secretion (Fig. 2A). The effect of AG on insulin secretion under hyperglycemic conditions was prevented by co-incubation with $1 \mu \mathrm{M}$ of an inverse agonist ([D-Arg1, D-Phe5, D-Trp7, 9, Leu11]-substance P) or an antagonist (GHRP-6 [D-Lys3]) of GHSR-1a (Fig. 2B). Incubation of pancreas islets with $10 \mathrm{nM}$ ghrelin (1-5) Amide [Dap3]-octanoyl, a pentapeptide that binds to GHSR-1a, caused a $57 \%$ decrease in insulin secretion that was similar to the effect of $0.1 \mathrm{nM} \mathrm{AG}$ (Fig. 2C). There was no significant difference between islets from male and from female pups in any of the experiments.

Effect of AG, UAG, and GHRP-6 [D-Lys3] administration on glucose homeostasis at birth. Maternal glucose, insulin, and glucagon concentrations in dams at delivery were 92 [2] $\mathrm{mg} / \mathrm{dL}, 1.1$ [0.2] ng/mL, and 109 [6] pg/mL, respectively. In control neonates, glucose concentrations decreased significantly between birth and 45 min and went up again by 180 min (ANOVA: $p<0.001$ ) while insulin concentrations were lower at 45 and 180 min compared with baseline (ANOVA: $p=0.001)$. Glucagon concentrations remained stable during the first 180 min of life (Fig. 3). Administration of AG, UAG, or GHRP-6 [D-Lys3], a GHSR-1a antagonist, did not affect glucose (Fig. 3A), insulin (Fig. 3B) or glucagon (Fig. 3C) concentrations at $45 \mathrm{~min}$ or $180 \mathrm{~min}$ of life (Fig. 3).

Effect of AG, UAG, ghrelin (1-5) amide [Dap3]-octanoyl or co-administration of ghrelin (1-5) amide, [Dap3]- 

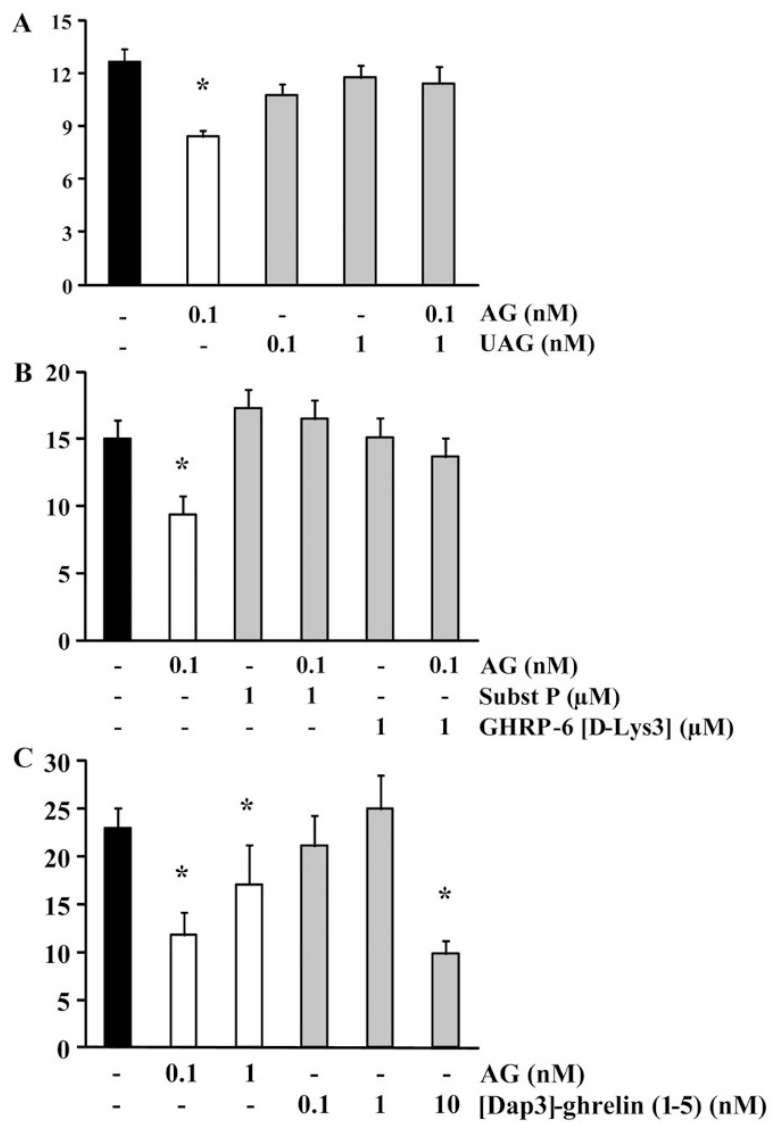

Figure 2. Effects of $(A)$ : unacylated ghrelin (UAG, $n=26-30 / g r) ;(B)$ : GHSR-1a antagonists (Lys GHRP-6) or inverse agonists (Subst P) $(n=$ $14-16 / g r) ; C$ : [DAP-3]-ghrelin (1-5) $(n=14-16 / g r)$ on insulin secretion by cultured pancreas islets from 5-d-old rat neonates under hyperglycemic (16.7 $\mathrm{mM}$ ) conditions. $\mathbf{\square}$, control; $\square$, AG only; $\square$, specific experimental conditions. One-way ANOVA: $A, p<0.001 ; B, p=0.044 ;(C), p=0.001 . * p<$ 0.05 compared with control group. Values are given in mean (SE). Subst P: [D-Arg1, D-Phe5, D-Trp7,9, Leu11]-substance P. [Dap3]-ghrelin (1-5): Ghrelin (1-5) Amide, [Dap3]-octanoyl.

octanoyl and GHRP-6 [D-Lys3] on glucose homeostasis in 6-d-old pups. Blood glucose and plasma insulin and C-peptide concentrations were similar in 6-d-old rat pups $20 \mathrm{~min}$ after the i.p. administration of $1.4 \mathrm{~g} / \mathrm{kg}$ of glucose or of glucose together with AG, UAG, ghrelin (1-5) amide [Dap3]-octanoyl or a mixture of ghrelin (1-5) amide [Dap3]-octanoyl and GHRP-6 [D-Lys3] (Table 1). The results were not influenced by gender.

\section{DISCUSSION}

This is the first in vitro demonstration in rat neonates that AG causes a marked decrease in insulin secretion from cultured pancreas islets. We also show that the ghrelin analogue ghrelin (1-5) amide [Dap3]-octanoyl has similar effects, although at concentrations that are 1-2 orders of magnitude greater than for AG. In contrast, in vivo, exogenous administration of AG in rat neonates at birth or at $6 \mathrm{~d}$ of age did not cause any detectable change in glucose homeostasis.

In vitro inhibition of insulin secretion by neonatal pancreas islets was observed for AG but not for UAG. Furthermore, the effect of AG was inhibited by an antagonist or an inverse
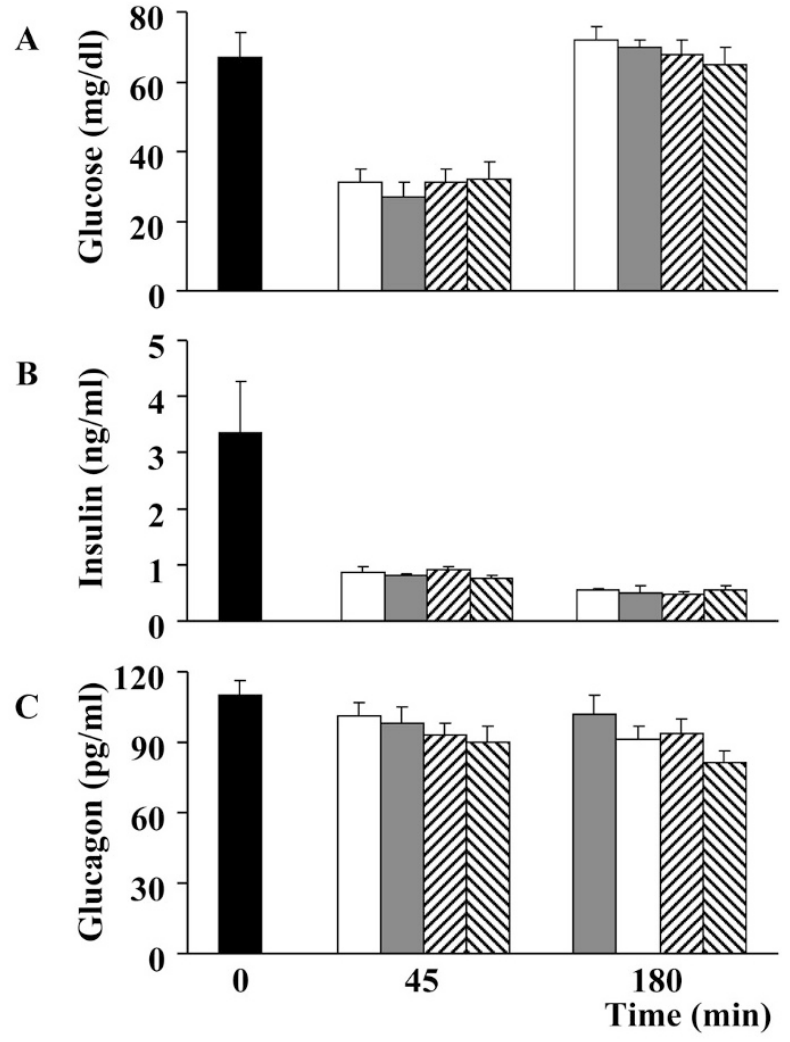

Figure 3. Effect of acylated ghrelin (AG), unacylated ghrelin (UAG), and GHRP-6 [D-Lys3] on $(A)$ glucose, $(B)$ insulin, and $(C)$ glucagon concentrations in rat neonates at birth $n=6-8$ /group and time point. $\square$, baseline; $\square$, control; $\square$, AG; $\mathbf{\nabla}$, UAG; $\boldsymbol{Q}$, GHRP-6 [D-Lys3]. Glucose-2-way ANOVA: time $p<0.001$; group: not significant. Insulin-2-way ANOVA: time $p<$ 0.001; group: not significant. Glucagon: not significant. Values are given in mean (SE)

agonist of GHSR-1a. These data suggest that, similar to adult animals, the effect of ghrelin on insulin secretion in rat neonates is mediated by GHSR-1a and that octanoylation of ghrelin is required (9). The insulinostatic effect of $A G$ was abolished by co-incubation with UAG at a 1 to 10 (AG to UAG) molar ratio. Several explanations have been proposed for this observation: competition of UAG with AG for GHSR-1a (15) (which seems unlikely as UAG does not bind GHSR-1a even at micromolar concentrations (11)) and stimulation of insulin release by UAG through nonGHSR-1a mechanisms (16). Furthermore, we show that in vitro, ghrelin (1-5) amide, [Dap3]-octanoyl, an octanoylated pentapeptide that includes the first 5 aminoacids of ghrelin (the minimal sequence required for binding to GHSR-1a) (11), also inhibits insulin secretion but at concentrations that are 1-2 orders of magnitude greater than for AG. This is consistent with in vitro data showing that the concentration of a similar pentapeptide required for $50 \%$ specific binding to the GHSR-1a is 200 times greater than for natural ghrelin (11).

We then investigated whether our in vitro findings could apply to in vivo situations and investigated the effect of exogenous administration of ghrelin in rat pups at birth and on the sixth day of life. The physiology of mammalian glucose homeostasis during the perinatal period is unique and markedly differs from adults. Birth is associated with instant dis- 
Table 1. In vivo effect of acylated ghrelin, unacylated ghrelin, [DAP-3]-ghrelin (1-5) and co-administration of [DAP-3]-ghrelin (1-5) and GHRP-6 [D-Lys3] on glucose, insulin, and C-peptide concentrations in 6-day-old rat neonates

\begin{tabular}{lccccc}
\hline & Glucose & AG & UAG & [Dap3]-ghrelin (1-5) & $\begin{array}{c}\text { [Dap3]-ghrelin (1-5) + } \\
\text { GHRP-6 [D-Lys3] }\end{array}$ \\
\hline Glucose (mg/dl) & $261(9)$ & $268(6)$ & $261(4)$ & $254(7)$ & $268(7)$ \\
Insulin $(\mathrm{ng} / \mathrm{ml})$ & $15.8(2.4)$ & $15.1(2.5)$ & $13.4(3.1)$ & $14.9(3.5)$ & $11.8(2.3)$ \\
C-peptide (ng/ml) & $22.2(1.1)$ & $21.4(1.1)$ & $20.2(1.5)$ & $21.1(1.8)$ & $19.8(1.6)$ \\
\hline
\end{tabular}

Values are given in mean (SE).

$n=16-20 /$ group.

AG, acylated ghrelin; UAG, unacylated ghrelin; [DAP-3]-ghrelin (1-5), Ghrelin (1-5) Amide, [Dap3]-Octanoyl.

continuation of maternal glucose as a source of carbohydrates. Maternal milk in rats contains little lactose and $60 \%$ of the calories originate from fat. Thus, conversion of fat into glucose is a key step in the maintenance of the glycemia in the neonate. This is achieved by the rapid development of hepatic neoglucogenesis and is associated with a decrease in insulin secretion and an increase in glucagon secretion (rev in Ref. 10). Consistent with these data, we observed a transient decrease in neonatal glycemia and a marked decrease in circulating insulin during the first $3 \mathrm{~h}$ of life, while glucagon concentrations were readily detectable (Fig. 3). However, in contrast to our hypothesis, AG administration did not appear to cause an earlier or higher increase in blood glucose in the first $3 \mathrm{~h}$ of life and did not affect insulin or glucagon concentrations. A potential explanation for our finding is that we did not investigate appropriate time points. However, in our experiment, blood glucose and insulin were measured first 45 min after the first injection, at a time when blood glucose was found to be at a nadir, and second at $180 \mathrm{~min}$ of life, following two consecutive AG injections, when blood glucose was found to have recovered. Taken together, our data do not support a major role for $\mathrm{AG}$ in the maintenance of neonatal glycemia at birth.

In contrast to our own unpublished findings in adult rats and to published data in adult mice (17), AG administration in 6-d-old pups did not affect glucose or insulin concentrations under hyperglycemic conditions. We chose to measure Cpeptide in addition to insulin to ensure that the lack of a measurable effect on systemic insulin concentrations was not masked by the degradation of portal insulin when crossing the liver (16). Indeed, the half-life of insulin is short (4-5 $\mathrm{min}$ in the adult rat (18)), and the liver takes up and degrades $50 \%$ of portal insulin at the first pass (19). In contrast, the concentrations of C-peptide, which is secreted in equimolar concentrations as insulin, are minimally affected by the liver and its half life is 5-10 times longer than insulin (20). Nevertheless, C-peptide concentrations were not affected by ghrelin.

Because of the rapid deacylation of AG in the neonate (14), which could explain the absence of demonstrable effect of AG on glucose homeostasis in vivo, we also administered ghrelin (1-5) amide, [Dap3]-octanoyl. This pentapeptide retains the capability of binding and activating the GHSR-1a (11) and has an amide bond between Ser3 of ghrelin and the octanoyl chain that makes this peptide more resistant to deacylation. Similar to ghrelin, ghrelin (1-5) amide, [Dap3]-octanoyl was shown to inhibit exocrine pancreas secretion in vivo (21). In contrast, it did not affect stomach emptying (22). In our in vitro experi- ments, ghrelin (1-5) amide, [Dap3]-octanoyl inhibited insulin secretion by the neonatal pancreas islet at a concentration of $10 \mathrm{nM}(1-2$ orders of magnitude greater than AG). In vivo, we chose a dose of ghrelin (1-5) amide, [Dap3]-octanoyl that was similar to the dose of AG. The rationale for this design was that in contrast to ghrelin (1-5) amide, [Dap3]-octanoyl, AG is degraded very quickly in young rats as recently shown by us (14), resulting in circulating AG concentrations that would be 1-2 orders of magnitude lower compared with the concentrations of ghrelin (1-5) amide, [Dap3]-octanoyl. However, we did not observe an effect of ghrelin (1-5) amide, [Dap3]octanoyl on glucose-stimulated insulin or C-peptide secretion in vivo.

The physiologic role of ghrelin in overall glucose homeostasis remains incompletely understood. Our in vitro demonstration that AG under normo or hyperglycemic conditions decreases insulin secretion in rat pups is consistent with data in adult rodents reported in most $(15,17,23,24)$ but not in all (25) studies. However, in vivo, the situation is more complex and administration of AG was shown to affect glucose homeostasis not only by decreasing glucose-stimulated insulin secretion (17) but also by decreasing insulin sensitivity (26). In addition, while the in vivo effects of $\mathrm{AG}$ on glucose homeostasis are usually attributed to a direct effect of the acylated form of ghrelin, it should be remembered that, in vivo, AG is rapidly deacylated into UAG (14), which has effects opposite to those of AG on glucose homeostasis. UAG may stimulate insulin secretion under hyperglycemic conditions (16) and increases insulin sensitivity (27). Studies performed in several animal models that overexpress ghrelin have attempted to clarify this situation. By using a transgenic mouse model that leads to $\mathrm{a} \approx 50 \%$ increase in circulating $\mathrm{AG}$ and $\mathrm{a} \approx 25 \%$ increase in UAG, Bewick et al. (28) observed a decrease in glucose-induced insulin secretion without changes in insulin sensitivity. In contrast, Zhang et al. (29), using a model that increases mainly UAG concentrations, observed an increase in insulin sensitivity. In the study by Iwakura $e t$ al. (30), in which both AG and UAG concentrations were markedly increased, glucose intolerance was found to be due to decreased insulin response to a glucose load, while insulin sensitivity was unaffected. Taken together, these data suggest that the ratio of AG to UAG, more than the absolute concentrations of AG, may regulate glucose homeostasis in vivo.

This hypothesis is relevant to perinatal rat physiology and may explain the absence of in vivo effects of $A G$ in our experiments. We recently demonstrated in vitro that AG degradation was much faster in rat neonates compared with adult 
rats (14). Although only 59\% of AG was transformed into UAG and non-UAG products over $30 \mathrm{~min}$ in adult animals, this percentage was $92 \%$ in rat neonates. As a consequence, the AG to UAG ratio at the end of the incubation was six times lower in rat neonates compared with adult rat. We speculate that the discrepancy between the in vitro effects of AG (similar in adult rats and in rat neonates) and the in vivo effects of $\mathrm{AG}$ (detectable in adult rats but not, in this study, during the perinatal period) may be due to a smaller AG to UAG ratio in the rat neonate that would counteract the effects of $A G$ on glucose homeostasis.

The AG to UAG ratio may also be relevant to perinatal physiology and to the treatment of Type 2 diabetes in humans. Insulin is an anabolic hormone and is necessary for growth and development, in particular during the perinatal period (31). We speculate that a faster degradation of AG and a lower AG to UAG ratio could prevent an inappropriate inhibition of insulin secretion by the pancreas islet and favor anabolism. From a clinical point of view, in subjects with Type 2 diabetes, a lower AG to UAG ratio may be of benefit by preventing inhibition of insulin release and increasing peripheral insulin sensitivity. This could be achieved by the development of inhibitors of the ghrelin O-acyl transferase, the enzyme that was recently shown to acylate proghrelin in tissues $(32,33)$.

In conclusion, our in vitro data show that perinatal islets, similar to adult islets, are sensitive to the insulinostatic effects of AG. However, in contrast to adult rodents, our data do not support our hypothesis that AG regulates insulin secretion in vivo during the perinatal period. This may be due to a faster degradation of AG compared with the adult animal and may represent a physiologic mechanism aiming at preserving the secretion of insulin, an anabolic hormone important for optimal growth and development.

Acknowledgments. The technical contribution from $\mathrm{Mr}$ Alfred CK Wong is gratefully acknowledged.

\section{REFERENCES}

1. Kojima M, Hosoda H, Date Y, Nakazato M, Matsuo H, Kangawa K 1999 Ghrelin is a growth-hormone-releasing acylated peptide from stomach. Nature 402:656-660

2. Shintani M, Ogawa Y, Ebihara K, Aizawa-Abe M, Miyanaga F, Takaya K, Hayashi T, Inoue G, Hosoda K, Kojima M, Kangawa K, Nakao K 2001 Ghrelin, an endogenous growth hormone secretagogue, is a novel orexigenic peptide that antagonizes leptin action through the activation of hypothalamic neuropeptide Y/Y1 receptor pathway. Diabetes 50:227-232

3. Kojima M, Kangawa K 2006 Drug insight: the functions of ghrelin and its potential as a multitherapeutic hormone. Nat Clin Pract Endocrinol Metab 2:80-88

4. Chanoine JP, Wong AC 2004 Ghrelin gene expression is markedly higher in fetal pancreas compared with fetal stomach: effect of maternal fasting. Endocrinology $145: 3813-3820$

5. Chanoine JP, Wong AC, Barrios V 2006 Obestatin, acylated and total ghrelin concentrations in the perinatal rat pancreas. Horm Res 66:81-88

6. Walia P, Asadi A, Kieffer TJ, Johnson JD, Chanoine JP 2009 Ontogeny of ghrelin, obestatin, preproghrelin, and prohormone convertases in rat pancreas and stomach. Pediatr Res 65:39-44

7. Kageyama H, Funahashi H, Hirayama M, Takenoya F, Kita T, Kato S, Sakurai J, Lee EY, Inoue S, Date Y, Nakazato M, Kangawa K, Shioda S 2005 Morphological analysis of ghrelin and its receptor distribution in the rat pancreas. Regul Pept 126:67-71

8. Dezaki K, Sone H, Yada T 2008 Ghrelin is a physiological regulator of insulin release in pancreatic islets and glucose homeostasis. Pharmacol Ther 118:239-249

9. Dezaki K, Hosoda H, Kakei M, Hashiguchi S, Watanabe M, Kangawa K, Yada T 2004 Endogenous ghrelin in pancreatic islets restricts insulin release by attenuating $\mathrm{Ca}^{2+}$ signaling in beta-cells: implication in the glycemic control in rodents. Diabetes 53:3142-3151

10. Girard J, Ferre P, Pegorier JP, Duee PH 1992 Adaptations of glucose and fatty acid metabolism during perinatal period and suckling-weaning transition. Physiol Rev 72:507-562

11. Bednarek MA, Feighner SD, Pong SS, McKee KK, Hreniuk DL, Silva MV, Warren VA, Howard AD, Van Der Ploeg LH, Heck JV 2000 Structure-function studies on the new growth hormone-releasing peptide, ghrelin: minimal sequence of ghrelin necessary for activation of growth hormone secretagogue receptor 1a. J Med Chem 43:4370-4376

12. Asakawa A, Inui A, Kaga T, Katsuura G, Fujimiya M, Fujino MA, Kasuga M 2003 Antagonism of ghrelin receptor reduces food intake and body weight gain in mice. Gut 52:947-952

13. Holst B, Cygankiewicz A, Jensen TH, Ankersen M, Schwartz TW 2003 High constitutive signaling of the ghrelin receptor-identification of a potent inverse agonist. Mol Endocrinol 17:2201-2210

14. Ni H, Walia P, Chanoine JP 2010 Ontogeny of acylated ghrelin degradation in the rat. Peptides 31:301-306

15. Salehi A, Dornonville de la Cour C, Hakanson R, Lundquist I 2004 Effects of ghrelin on insulin and glucagon secretion: a study of isolated pancreatic islets and intact mice. Regul Pept 118:143-150

16. Gauna C, Kiewiet RM, Janssen JA, van de Zande B, Delhanty PJ, Ghigo E, Hofland LJ, Themmen AP, van der Lely AJ 2007 Unacylated ghrelin acts as a potent insulin secretagogue in glucose-stimulated conditions. Am J Physiol Endocrinol Metab 293:E697-E704

17. Reimer MK, Pacini G, Ahren B 2003 Dose-dependent inhibition by ghrelin of insulin secretion in the mouse. Endocrinology 144:916-921

18. Canas X, Fernandez-Lopez JA, Ardevol A, Adan C, Esteve M, Rafecas I, Remesa X, Alemany M 1995 Rat insulin turnover in vivo. Endocrinology 136:3871-3876

19. Duckworth WC, Bennett RG, Hamel FG 1998 Insulin degradation: progress and potential. Endocr Rev 19:608-624

20. Marques RG, Fontaine MJ, Rogers J 2004 C-peptide: much more than a byproduct of insulin biosynthesis. Pancreas 29:231-238

21. Kapica M, Laubitz D, Puzio I, Jankowska A, Zabielski R 2006 The ghrelin pentapeptide inhibits the secretion of pancreatic juice in rats. J Physiol Pharmacol 57:691-700

22. Dornonville de la Cour C, Lindstrom E, Norlen P, Hakanson R 2004 Ghrelin stimulates gastric emptying but is without effect on acid secretion and gastric endocrine cells. Regul Pept 120:23-32

23. Colombo M, Gregersen S, Xiao J, Hermansen K 2003 Effects of ghrelin and other neuropeptides (CART, MCH, Orexin A and B, and GLP-1) on the release of insulin from isolated rat islets. Pancreas 27:161-166

24. Qader SS, Lundquist I, Ekelund M, Hakanson R, Salehi A 2005 Ghrelin activates neuronal constitutive nitric oxide synthase in pancreatic islet cells while inhibiting insulin release and stimulating glucagon release. Regul Pept 128:51-56

25. Date Y, Nakazato M, Hashiguchi S, Dezaki K, Mondal MS, Hosoda H, Kojima M, Kangawa K, Arima T, Matsuo H, Yada T, Matsukura S 2002 Ghrelin is present in pancreatic alpha-cells of humans and rats and stimulates insulin secretion. Diabetes 51:124-129

26. Sun Y, Asnicar M, Saha PK, Chan L, Smith RG 2006 Ablation of ghrelin improves the diabetic but not obese phenotype of ob/ob mice. Cell Metab 3:379-386

27. Iwakura H, Hosoda K, Son C, Fujikura J, Tomita T, Noguchi M, Ariyasu H, Takaya K, Masuzaki H, Ogawa Y, Hayashi T, Inoue G, Akamizu T, Hosoda H, Kojima M, Itoh H, Toyokuni S, Kangawa K, Nakao K 2005 Analysis of rat insulin II promoterghrelin transgenic mice and rat glucagon promoter-ghrelin transgenic mice. J Biol Chem 280:15247-15256

28. Bewick GA, Kent A, Campbell D, Patterson M, Ghatei MA, Bloom SR, Gardiner JV 2009 Mice with hyperghrelinemia are hyperphagic and glucose intolerant and have reduced leptin sensitivity. Diabetes 58:840-846

29. Zhang W, Chai B, Li JY, Wang H, Mulholland MW 2008 Effect of des-acyl ghrelin on adiposity and glucose metabolism. Endocrinology 149:4710-4716

30. Iwakura H, Ariyasu H, Li Y, Kanamoto N, Bando M, Yamada G, Hosoda H, Hosoda K, Shimatsu A, Nakao K, Kangawa K, Akamizu T 2009 A mouse model of ghrelinoma exhibited activated growth hormone-insulin-like growth factor I axis and glucose intolerance. Am J Physiol Endocrinol Metab 297:E802-E811

31. Hill DJ, Milner RD 1985 Insulin as a growth factor. Pediatr Res 19:879-886

32. Gutierrez JA, Solenberg PJ, Perkins DR, Willency JA, Knierman MD, Jin Z, Witcher DR, Luo S, Onyia JE, Hale JE 2008 Ghrelin octanoylation mediated by an orphan lipid transferase. Proc Natl Acad Sci U S A 105:6320-6325

33. Yang J, Brown MS, Liang G, Grishin NV, Goldstein JL 2008 Identification of the acyltransferase that octanoylates ghrelin, an appetite-stimulating peptide hormone. Cell 132:387-396 